classification is a prognostic indicator in thymoma. Ann Thorac Surg 2004;77:1183-8.

6. Shelly S, Agmon-Levin N, Altman A, Shoenfeld Y. Thymoma and autoimmunity. Cell Mol Immunol 2011;8:199-202.

7. Thompson CA, Steensma DP. Pure red cell aplasia associated with thymoma: clinical insights from a 50-year single-institution experience. Br J Haematol 2006;135:405-7.

8. Maslovsky I, Gefel D, Uriev L, Ben Dor D, Lugassy G. Malignant thymoma complicated by amegakaryocytic thrombocytopenic purpura. Eur J Intern Med 2005;16:523-4.

9. Kobayashi H, Kitano K, Ishida F, et al. Aplastic anemia and idiopathic thrombocytopenic purpura with antibody to platelet glycoprotein IIb/IIIa following resection of malignant thymoma. Acta Haematol 1993;90:42-5.

10. Bussel JB, Cheng G, Saleh MN, et al. Eltrombopag for the treatment of chronic idiopathic thrombocytopenic purpura. N Engl J Med 2007;357:2237-47.

11. Cheng G, Saleh MN, Marcher C, et al. Eltrombopag for management of chronic immune thrombocytopenia (RAISE): a 6-month, randomised, phase 3 study. Lancet 2011;377:393-402.

\section{Coexistence of BCR/ABL 1-positive chronic myeloid leukemia and JAK2 V617F-mutated myelofibrosis successfully treated with dasatinib and ruxolitinib}

TO THE EDITOR: Myeloproliferative neoplasms (MPNs) are clonal hematopoietic stem cell disorders characterized by the proliferation of cells of one or more of the myeloid lineages. Depending on the presence or absence of a $B C R / A B L 1$ translocation, they can be divided into $B C R / A B L 1$-positive chronic myeloid leukemias (CMLs) and $B C R / A B L 1$-negative MPNs, respectively [1]. Thus, by World Health Organization (WHO) criteria, primary myeloid fibrosis (PMF) cannot coexist with CML, as the exclusion of $\mathrm{CML}$ is one of the major criteria for PMF diagnosis. However, there are a few reports that have described the coexistence of the two diseases [2-4]. Herein, we report the case of a patient who initially presented with features of CML and PMF. He was treated with dasatinib, and soon after experienced aggravated constitutional symptoms as well as leukocytosis. Concomitant administration of ruxolitinib ameliorated his symptoms.

The 68-year-old man had visited our clinic with hematologic test abnormalities; that is, $28,710 / \mu \mathrm{L}$ for white blood cell (WBC) counts, $10.4 \mathrm{~g} / \mathrm{dL}$ for hemoglobin, and $244,000 / \mu \mathrm{L}$ for platelet counts. A peripheral blood smear examination demonstrated an excess of myelocytes and metamyelocytes. Elevation of the serum lactate dehydrogenase (LD) level was also noted. His abdominal computed tomography (CT) scan demonstrated marked splenomegaly $(22.7 \mathrm{~cm})$.

Suspecting an MPN of mostly CML, a bone marrow examination was done. The cellularity was almost $100 \%$ and prominent granulopoiesis was noted. The megakaryocytes were high in number, and some of them showed atypia, such as decreased cytoplasm, cloud-like nuclei, and dense cluster formation (Fig. 1). A grade I-II fibrosis was observed. Regarding the cytogenetics, a $B C R / A B L 1$ translocation was observed in 9 out of $21(42.8 \%)$ cells by chromosomal analysis and in 246 out of 400 (61.5\%) cells by fluorescence in situ hybridization (FISH). The transcript level determined by real-time quantitative polymerase chain reaction (RQ-PCR) was $65.7 \%$ IS (international scale). In addition, a JAK2-V617F mutation was detected. These findings were sufficient for the diagnosis of CML. The patient was started on dasatinib $100 \mathrm{mg}$ once daily on May 13, 2016. During the first 3 months of treatment, the patient had never achieved a complete hematologic response, as the leukocyte counts remained at around $15,000 / \mu \mathrm{L}$.

In the bone marrow examination done at 3 months, atypical megakaryocytes were still noted as well as grade II fibrosis. A CT scan revealed the spleen size to be $20.9 \mathrm{~cm}$. Suspecting treatment failure, the dasatinib was increased to $140 \mathrm{mg}$ once daily. Five days later, a complete cytogenetic response test reported none of the 14 cells by chromosomal analysis and none of the 400 cells by FISH had the $B C R / A B L 1$ translocation. A major molecular response was also achieved $(0.07 \%$ IS). When the patient visited the clinic 2 weeks later, he complained of constitutional symptoms, such as fever, poor oral intake, headache, and severe fatigue. Unexpected leukocytosis $(37,860 / \mu \mathrm{L})$ and anemia $(7.9 \mathrm{~g} / \mathrm{dL})$ were noted. He was started on hydroxyurea to ameliorate his leukocytosis, and the dasatinib was reduced to $100 \mathrm{mg}$ once daily. Two weeks later, his WBC count had decreased and his symptoms also improved. Because hydroxyurea worsened his anemia, he had taken it temporarily, and when he stopped it, the WBC count increased. At the 6-month evaluation, the bone marrow findings were not significantly different from the previous findings. The spleen size had decreased slightly to $18.8 \mathrm{~cm}$. Complete cytogenetic and molecular $(0.00 \%$ IS) responses were also sustained. Despite these responses, the patient had been dependent on hydroxyurea for control of the symptoms and leukocytosis.

We assumed that both CML and PMF clones existed, and suppressing the CML clones may have stimulated the PMF clone, which resulted in leukocytosis and aggravation of the constitutional symptoms. Thus, on December 9, 2016, the patient started to take ruxolitinib $20 \mathrm{mg}$ twice daily concurrently with dasatinib. At this time, his MPN10 score was 62, and the international prognostic scoring system (IPSS) group was high-risk owing to his age, symptoms, and leukocytosis. The leukocytosis resolved after 2 weeks, and so did the constitutional symptoms. In March 2017, after 3 months of ruxolitinib treatment, the MPN10 score improved to 26 and the spleen size decreased to $16.7 \mathrm{~cm}$. 


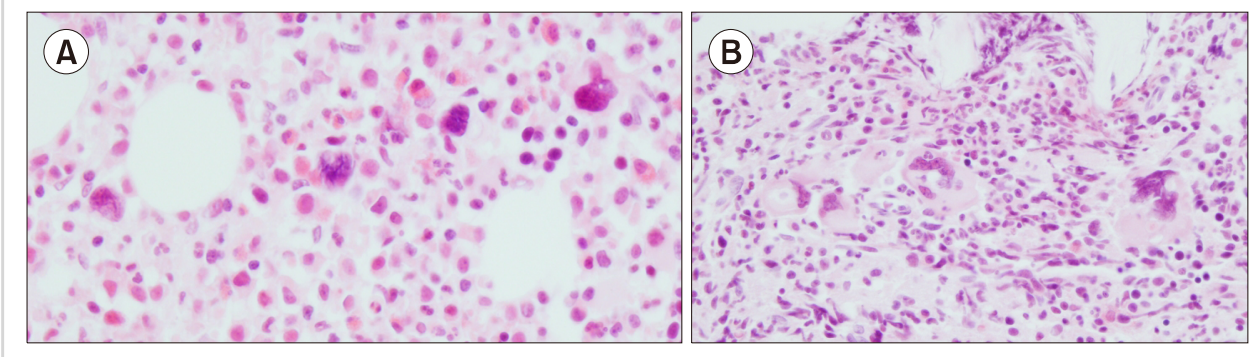

Fig. 1. Bone marrow biopsy specimens at the time of diagnosis (hematoxylin and eosin stain, $\times 400$ ). Atypical megakaryocytes showing cloud-like nuclei with a high nucleusto-cytoplasm ratio (A). Denseclusters of megakaryocytes (B).

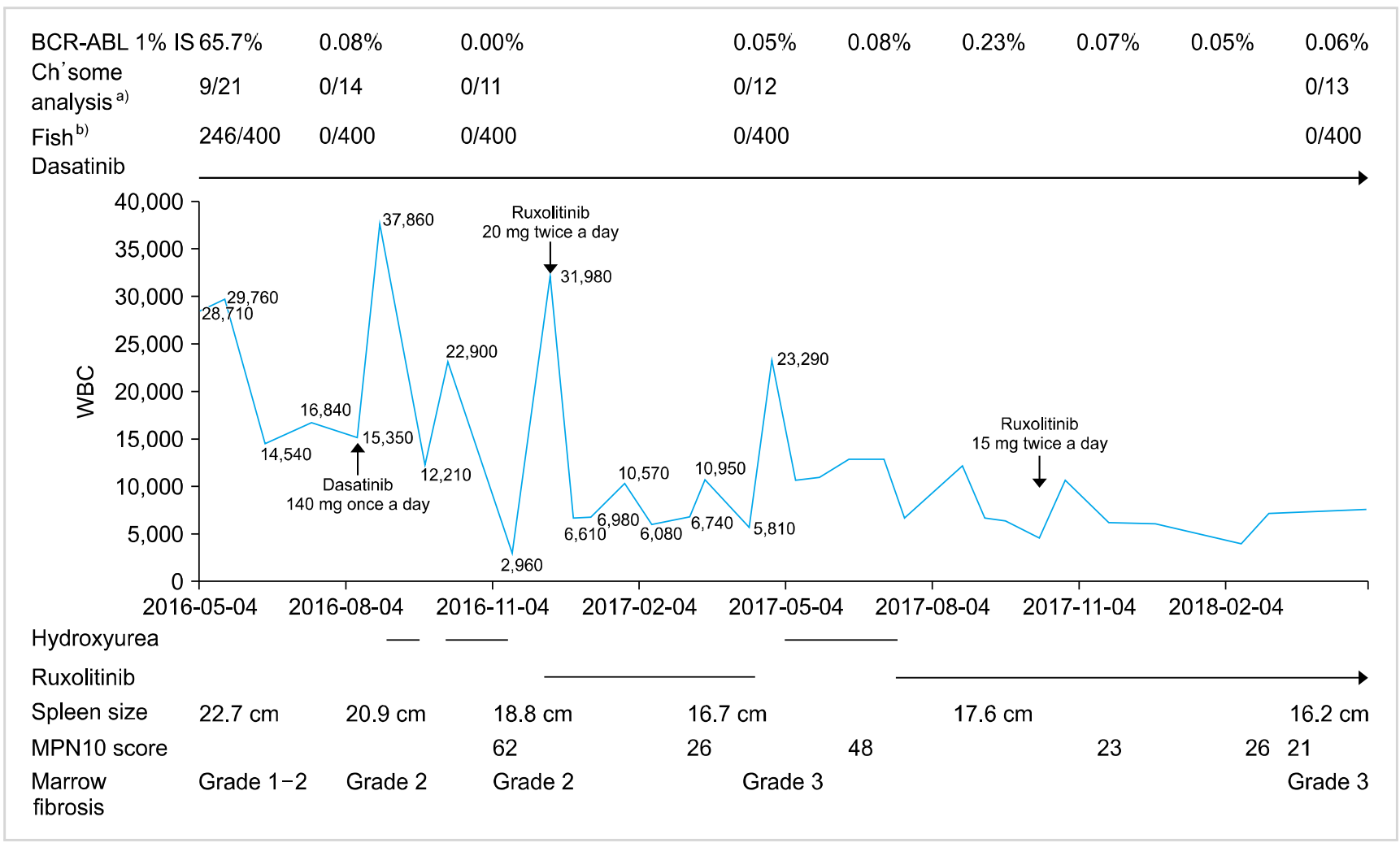

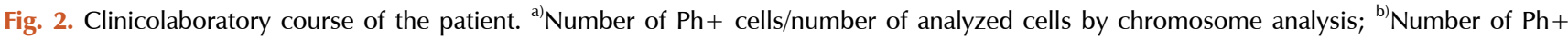
cells/number of analyzed cells by fluorescence in situ hybridization analysis. $\mathrm{Ph}+$, Philadelphia chromosome-positive.

The IPSS grouping was intermediate-1, owing to his age. After 12 months of dasatinib treatment, a bone marrow examination carried out in May 2017 demonstrated still-noted hypercellularity, atypical megakaryocytes, and grade III myelofibrosis. Continuing complete cytogenetic and molecular responses were observed.

The patient continued to take dasatinib and ruxolitinib until April 2017. He then stopped taking ruxolitinib because of an insurance issue, whereupon the constitutional symptoms and leukocytosis recurred. He resumed the concurrent treatment in July 2017 and the dose of ruxolitinib was decreased to $15 \mathrm{mg}$ twice daily because of anemia and thrombocytopenia. He has been taking both drugs for 18 months while maintaining the major molecular response and improvement in MPN10 score of over 50\%. In May 2018, 2-year follow-up studies were performed. The tran- script level determined by RQ-PCR was $0.06 \%$ IS, and the spleen size had decreased to $16.2 \mathrm{~cm}$. The IPSS grouping was intermediate-1. Bone marrow examination showed grade III myelofibrosis with increased fibroblasts. The clinicolaboratory course is described in Fig. 2.

We have described the case of a patient who initially presented the features of both CML and PMF. After taking dasatinib, he promptly achieved cytogenetic and molecular responses but not a hematologic response. Moreover, increasing the dose of dasatinib induced aggravated leukocytosis and constitutional symptoms. Despite the sustained molecular response, the persistent leukocytosis and constitutional symptoms bothered the patient, but these resolved after he started taking ruxolitinib. He has been faring well for 18 months with the concurrent treatment.

According to the WHO classification, the diagnosis of 
CML and that of other $B C R / A B L 1$-negative MPNs are mutually exclusive. However, there are several reports of the synchronous existence of both CML and PMF [2-4], or polycythemia vera $[5,6]$. The common findings of these reports were manifestations of MPNs (myelofibrosis, development of constitutional symptoms, or erythrocytosis) and an increase of $J A K 2$ burden that occurred after treatment with a $B C R / A B L 1$ tyrosine kinase inhibitor (TKI).

In our case, the patient was definitively diagnosed with CML as he presented with granulocytosis, an excess of precursors, and the presence of $B C R / A B L 1$ translocation. At the same time, he also presented with several features favoring the diagnosis of PMF. First, an initial bone marrow examination showed fibrosis, and the megakaryocytes were large with cellular atypia typically found in PMF. Second, the patient had a $J A K 2$ mutation, which is not a common finding in patients with CML $[7,8]$. Third, only 9 out of 21 cells harbored the $B C R / A B L 1$ translocation according to chromosomal analysis, suggesting the existence of another clone. The subsequent findings after dasatinib treatment further confirmed that there were two clones competing with each other. Despite the prompt cytogenetic and molecular responses, the patient failed to achieve a hematologic response. Moreover, increasing the dasatinib dose aggravated the leukocytosis and constitutional symptoms, for which hydroxyurea was required. In addition, the progression of fibrosis was not impeded. It remains to be elucidated how these distinct clones developed. There are several cases of CML developing after the long-term treatment of MPNs [9, 10]. One hypothesis was that the first hit was a $J A K 2$ mutation, and constitutional $J A K 2$-mediated signals may have contributed to $B C R / A B L 1$-mutated clonal evolution.

Ruxolitinib is a potent JAK inhibitor, and its major role in $\mathrm{PMF}$ is for symptom control. Thus, its incorporation into $B C R / A B L 1$ TKI treatment is challenging as it also worsens anemia or thrombocytopenia, which was also the reason we had to reduce the dose for our patient.

Our experience provides several clinical implications. First, CML and other types of MPNs may coexist, even though the incidence may be very rare. Second, physicians should consider hidden MPNs when there are discrepancies in the molecular response and clinical response in CML. Third, although dasatinib and ruxolitinib can be safely combined, hematologic adverse events may be a concern. This is one of the few case reports of a patient who initially had features of both CML and PMF, and to the best of our knowledge, the first case report of treating such a patient with dasatinib and ruxolitinib.

Jun Ho $\mathrm{Yi}^{1}$, Hye Ryoun $\mathrm{Kim}^{2}$

${ }^{1}$ Division of Hematology-Oncology, Department of Medicine, ${ }^{2}$ Department of Laboratory Medicine,
Chung-Ang University, Seoul, Korea

Correspondence to: Jun Ho Yi

Division of Hematology-Oncology, Department of Medicine, Chung-Ang University, Heuksuk-ro 102, Dongjak-gu, Seoul 06973, Korea E-mail: xuno@cau.ac.kr

Received on Jul. 10, 2018; Revised on Oct. 18, 2018; Accepted on Oct. 29, 2018 https://doi.org/10.5045/br.2019.54.1.77

\section{Acknowledgments}

This study was supported by the Chung-Ang University Research Grants in 2017.

\section{Authors' Disclosures of Potential Conflicts of Interest}

No potential conflicts of interest relevant to this article were reported.

\section{REFERENCES}

1. Swerdlow SH, Campo E, Harris NL, et al, eds. WHO classification of tumours of haematopoietic and lymphoid tissues. Revised 4th ed. Lyon, France: IARC Press, 2017.

2. Hussein K, Bock O, Seegers A, et al. Myelofibrosis evolving during imatinib treatment of a chronic myeloproliferative disease with coexisting BCR-ABL translocation and JAK2V617F mutation. Blood 2007;109:4106-7.

3. Krämer A, Reiter A, Kruth J, et al. JAK2-V617F mutation in a patient with Philadelphia-chromosome-positive chronic myeloid leukaemia. Lancet Oncol 2007;8:658-60.

4. Bornhäuser M, Mohr B, Oelschlaegel U, et al. Concurrent JAK2(V617F) mutation and BCR-ABL translocation within committed myeloid progenitors in myelofibrosis. Leukemia 2007;21:1824-6.

5. Inami M, Inokuchi K, Okabe M, et al. Polycythemia associated with the JAK2V617F mutation emerged during treatment of chronic myelogenous leukemia. Leukemia 2007;21:1103-4.

6. Cambier N, Renneville A, Cazaentre T, et al. JAK2V617F-positive polycythemia vera and Philadelphia chromosome-positive chronic myeloid leukemia: one patient with two distinct myeloproliferative disorders. Leukemia 2008;22:1454-5.

7. Makishima H, Jankowska AM, McDevitt MA, et al. CBL, CBLB, TET2, ASXL1, and IDH1/2 mutations and additional chromosomal aberrations constitute molecular events in chronic myelogenous leukemia. Blood 2011;117:e198-206.

8. Pieri L, Spolverini A, Scappini B, et al. Concomitant occurrence of BCR-ABL and JAK2V617F mutation. Blood 2011;118:3445-6.

9. Zhou A, Knoche EM, Engle EK, Fisher DA, Oh ST. Concomitant JAK2 V617F-positive polycythemia vera and BCR-ABL-positive chronic myelogenous leukemia treated with ruxolitinib and dasatinib. Blood Cancer J 2015;5:e351.

10. Iurlo A, Gianelli U, Rapezzi D, et al. Imatinib and ruxolitinib association: first experience in two patients. Haematologica 2014; 99:e76-7. 